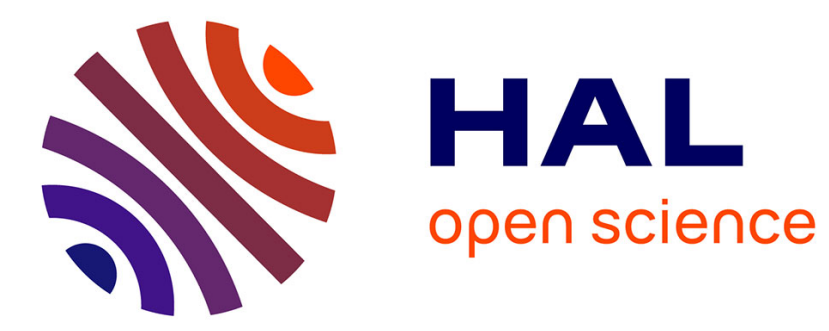

\title{
Lattice reorientation in tetragonal PMN-PT thin film induced by focused ion beam preparation for transmission electron microscopy
}

Thibaud Denneulin, Wanjoo Maeng, Chang-Beom Eom, Martin Hÿtch

\section{To cite this version:}

Thibaud Denneulin, Wanjoo Maeng, Chang-Beom Eom, Martin Hÿtch. Lattice reorientation in tetragonal PMN-PT thin film induced by focused ion beam preparation for transmission electron microscopy. Journal of Applied Physics, 2017, 121 (5), pp.055302. 10.1063/1.4975114 . hal-01707818

\author{
HAL Id: hal-01707818 \\ https://hal.science/hal-01707818
}

Submitted on 23 Feb 2018

HAL is a multi-disciplinary open access archive for the deposit and dissemination of scientific research documents, whether they are published or not. The documents may come from teaching and research institutions in France or abroad, or from public or private research centers.
L'archive ouverte pluridisciplinaire HAL, est destinée au dépôt et à la diffusion de documents scientifiques de niveau recherche, publiés ou non, émanant des établissements d'enseignement et de recherche français ou étrangers, des laboratoires publics ou privés. 


\section{Lattice reorientation in tetragonal PMN-PT thin film induced by focused ion beam preparation for transmission electron microscopy}

Thibaud Denneulin, Wanjoo Maeng, Chang-Beom Eom, and Martin Hÿtch

Citation: Journal of Applied Physics 121, 055302 (2017); doi: 10.1063/1.4975114

View online: https://doi.org/10.1063/1.4975114

View Table of Contents: http://aip.scitation.org/toc/jap/121/5

Published by the American Institute of Physics

\section{Articles you may be interested in}

Reversible transition between coherently strained $\mathrm{BiFeO}_{3}$ and the metastable pseudotetragonal phase on

$\left(\mathrm{LaAlO}_{3}\right)_{0.3}\left(\mathrm{Sr}_{2} \mathrm{AlTaO}_{6}\right)_{0.7}(001)$

Journal of Applied Physics 121, 054102 (2017); 10.1063/1.4975342

Ferroelectric, pyroelectric, and piezoelectric properties of a photovoltaic perovskite oxide Applied Physics Letters 110, 063903 (2017); 10.1063/1.4974735

Raman-strain relations in highly strained Ge: Uniaxial $\langle 100\rangle,\langle 110\rangle$ and biaxial (001) stress Journal of Applied Physics 121, 055702 (2017); 10.1063/1.4974202

Ultrahigh strain and piezoelectric behavior in relaxor based ferroelectric single crystals Journal of Applied Physics 82, 1804 (1997); 10.1063/1.365983

Theoretical analysis of a Love wave biosensor in liquid with a viscoelastic wave guiding layer Journal of Applied Physics 121, 054501 (2017); 10.1063/1.4975112

Laterally coupled distributed feedback lasers emitting at $2 \mu \mathrm{m}$ with quantum dash active region and high-dutycycle etched semiconductor gratings Journal of Applied Physics 121, 053101 (2017); 10.1063/1.4975036

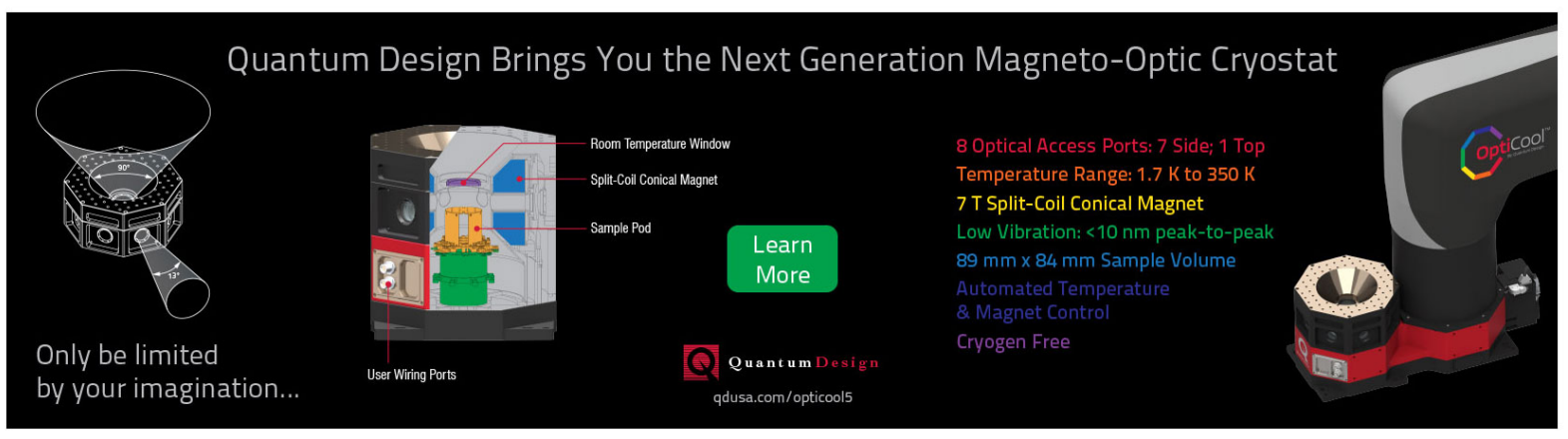




\title{
Lattice reorientation in tetragonal PMN-PT thin film induced by focused ion beam preparation for transmission electron microscopy
}

\author{
Thibaud Denneulin, ${ }^{1, a)}$ Wanjoo Maeng, ${ }^{2}$ Chang-Beom Eom, ${ }^{2}$ and Martin Hÿtch ${ }^{1}$ \\ ${ }^{1}$ CEMES, CNRS, 29 rue Jeanne Marvig, 31055 Toulouse, France \\ ${ }^{2}$ Department of Materials Science and Engineering, University of Wisconsin-Madison, Madison, \\ Wisconsin 53706, USA
}

(Received 6 October 2016; accepted 17 January 2017; published online 2 February 2017)

\begin{abstract}
Focused ion beam sample preparation for transmission electron microscopy (TEM) can induce relaxation mechanisms in epitaxial thin films. Here, we describe a relaxation mechanism that can occur in materials having a tetragonal structure. We investigated the lattice structure of a $600 \mathrm{~nm}$ thick $0.4\left[\mathrm{~Pb}\left(\mathrm{Mg}_{1 / 3} \mathrm{Nb}_{2 / 3}\right) \mathrm{O}_{3}\right]-0.6\left[\mathrm{PbTiO}_{3}\right]$ layer grown by epitaxy on (110) $\mathrm{GdScO}_{3}$ substrate using geometrical phase analysis applied to high resolution TEM images. The lattice mismatch at the interface is expected to favor the formation of $c$-domains. However, it was measured that the out-of-plane lattice parameter can decrease abruptly along the growth direction and the transition depends on the thickness of the TEM lamella. Different observations indicate that the crystal flipped by $90^{\circ}$ following the preparation of the sample, so that the $c$-axis is oriented in the thinning direction. Such a mechanism can easily lead to misinterpretations and might happen in other materials with a similar structure. Published by AIP Publishing.
\end{abstract}

[http://dx.doi.org/10.1063/1.4975114]

\section{INTRODUCTION}

Different sample preparation methods exist for transmission electron microscopy (TEM), such as focused ion beam (FIB), ${ }_{1}^{1}$ mechanical polishing, ${ }^{2}$ or small angle cleavage. ${ }^{3}$ Each technique has its own advantages and disadvantages. FIB is particularly convenient as it provides site specificity, consumes very small amounts of material, and can be automated. On the other hand, the ion beam can create damaged (amorphized) layers on the sides of the lamella which thickness depends on the beam energy and the angle of incidence., ${ }^{4,5}$

A problem that is common to all preparation techniques is the elastic relaxation occurring at the free surfaces of the thin foil, in particular, in epitaxial samples. ${ }^{6,7}$ It induces a bending of the lattice planes, which is responsible for strong diffraction contrast in bright-field or dark-field images, ${ }^{8}$ splitting of the high order Laue zone (HOLZ) lines in convergent beam electron diffraction (CBED), ${ }^{9,10}$ and also affect the contrast in high resolution images (HRTEM). ${ }^{11}$

Here, we report a relaxation mechanism observed after FIB preparation of an epitaxial layer of a tetragonal perovskite. It consists in a reorientation of the lattice structure, which allows the $c$-axis to flip from the growth direction to the thinning direction. The investigation was carried out on a $0.4\left[\mathrm{~Pb}\left(\mathrm{Mg}_{1 / 3} \mathrm{Nb}_{2 / 3}\right) \mathrm{O}_{3}\right]-0.6\left[\mathrm{PbTiO}_{3}\right](\mathrm{PMN}-\mathrm{PT})$ layer using HRTEM and geometrical phase analysis (GPA). ${ }^{12}$

PMN-PT materials are used in different types of piezoelectric transducers and actuators because of their very high electromechanical specifications. ${ }^{13}$ They can be used to produce ultrasounds for applications in medical imaging. ${ }^{14}$ Recently, they have attracted interest in microelectronics for the construction of a piezoelectronic transistor. ${ }^{15,16}$ Therefore,

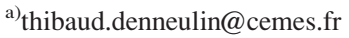

there is a growing need for the characterization of PMN-PT samples by TEM. However, it has been reported that the domain structure ${ }^{17}$ and the composition ${ }^{18}$ of these samples are quite sensitive to the preparation conditions.

\section{EXPERIMENTAL DETAILS}

The sample is a $600 \mathrm{~nm}$ thick layer with nominal composition 0.4PMN-0.6PT epitaxially grown by off-axis radio-frequency magnetron sputtering ${ }^{19}$ on $(110)_{\text {ortho }} \mathrm{GdScO}_{3}$ (GSO) substrate with a $10 \mathrm{~nm}$ thick $\mathrm{SrRuO}_{3}$ bottom electrode. ${ }^{20}$ In the relaxed state, at room temperature, $0.4 \mathrm{PMN}-0.6 \mathrm{PT}$ is expected to be tetragonal with lattice parameters $a=b=0.397 \mathrm{~nm}$ and $c=0.407 \mathrm{~nm}^{21,22} \mathrm{GdScO}_{3}$ and $\mathrm{SrRuO}_{3}$ are orthorhombic with lattice parameters $a=0.548 \mathrm{~nm}, b=0.575 \mathrm{~nm}, c=0.793 \mathrm{~nm}$ (Ref. 23) and $a=0.557 \mathrm{~nm}, b=0.553 \mathrm{~nm}, c=0.785 \mathrm{~nm},{ }^{24}$ respectively.

The sample was prepared as a parallel-sided cross-section thin foil using a focused ion beam and scanning electron microscope (FIB-SEM) FEI Helios platform. The sample was covered with electron beam deposited platinum $(\simeq 300 \mathrm{~nm}$ thick) and then ion beam deposited platinum ( $\simeq 3 \mu \mathrm{m}$ thick). The final milling was carried out at $8 \mathrm{kV}$ with a tilt angle of $\pm 2^{\circ}$ to compensate for the Gaussian shape of the ion beam. The use of this intermediate FIB operating voltage provides a compromise between reducing the surface damage and controlling the shape of the lamella (minimizing thickness variations), which is preferred for strain mapping over large areas.

HRTEM was carried out using two transmission electron microscopes. A Hitachi HF-3300 (I2TEM-Toulouse) equipped with a cold-field emission gun operated at $300 \mathrm{kV}$, an image corrector (CEOS B-COR for correcting off-axial aberrations), and a $4 \mathrm{k} \times 4 \mathrm{k}$ CCD camera (USC4000 Gatan) were used to acquire HRTEM images with a large field of view 
$(\approx 600 \mathrm{~nm})$. A FEI Tecnai F20 (SACTEM-Toulouse) equipped with a Schottky field emission gun operated at $200 \mathrm{kV}$, an image corrector, and a $2 \mathrm{k} \times 2 \mathrm{k}$ CCD camera were used to acquire complementary images.

The images were processed using the GPA 4.6 software (HREM Research, Inc.), a plug-in for Digital Micrograph (Gatan). Phase images were reconstructed using a halfcosine mask in Fourier space and were corrected for distortions introduced by the projector lens system and the camera. ${ }^{25}$ Deformation maps were obtained by taking the gradient of the phase image in the direction of the reciprocal lattice vector. The $\varepsilon_{z z}$ out-of-plane and $\varepsilon_{x x}$ in-plane deformation maps were calculated with the (001) and (100) PMN-PT reflections, respectively. If the $\mathrm{GdScO}_{3}$ substrate is within the field of view, the corresponding (110) ortho and (002) ortho reflections, respectively, were included in the mask.

\section{RESULTS}

Fig. 1(a) shows a SEM image of the sample prepared by FIB with parallel sides and two different lamella thicknesses. The thicknesses were estimated to be $80 \mathrm{~nm}$ and $140 \mathrm{~nm}$ $( \pm 20 \mathrm{~nm})$ from the top view image in Fig. 1(b). Figs. 1(c) and 1(d) show TEM images of the thin and thick parts of the lamella (observed along the [1 10$]$ orthorhombic axis of the GSO) which contain distinct features.

In the thin part (Fig. 1(c)), the PMN-PT layer can be separated into three different regions along the growth direction. The middle region (transition area) contains horizontal lines, which are reminiscent of the contrast of stacking faults or moiré patterns. The top and bottom regions are quite similar and show variations in contrast typical of local defects, bending of the crystal or $180^{\circ}$ domains. The top region will be called the FIB-induced area and this will be explained further with the deformation measurements.

The thick part of the lamella (Fig. 1(d)) contains needleshaped $a$-domains (or $90^{\circ}$ domains) where the $c$ parameter is in-plane. The $a / c$ domain walls are inclined by $45^{\circ}$ relatively to the bottom interface, following the (101) and $(-101)$ lattice planes. The formation of $a$-domains is a relaxation mechanism frequently observed in tetragonal perovskite thin films such as $\mathrm{PbZr}_{x} \mathrm{Ti}_{1-x} \mathrm{O}_{3}$ (with $x<0.5$ ), ${ }^{26,27}$ which allows the modulation of the average in-plane parameter. However, the domain walls appear more diffuse and the inclination changes with respect to the $\{101\}$ planes in a small region ( $\approx 60 \mathrm{~nm}$ thick) just below the surface. This region is indicated again as a FIB-induced area.

Fig. 2(a) shows an HRTEM image acquired in the thin part of the lamella with a large field view $(\approx 600 \mathrm{~nm}$, acquired with the I2TEM microscope). The sample was oriented slightly away from the zone-axis, along the (001) systematic row of the PMN-PT in order to increase the signal of the horizontal lattice planes. Fig. 2(b) is the $\varepsilon_{z z}$ out-of-plane deformation map obtained by GPA. The deformation is defined relatively to the GSO substrate $\left(\varepsilon=\left(d_{\mathrm{PMN}-\mathrm{PT}}-d_{\mathrm{GSO}}\right) / d_{\mathrm{GSO}}\right.$ with $d$ being the interplanar distance). Figs. 2(c) and 2(d) show a HRTEM image with the sample tilted along the (100) systematic row of the PMN-PT and the corresponding $\varepsilon_{x x}$ inplane deformation map. Finally, Fig. 2(e) shows profiles
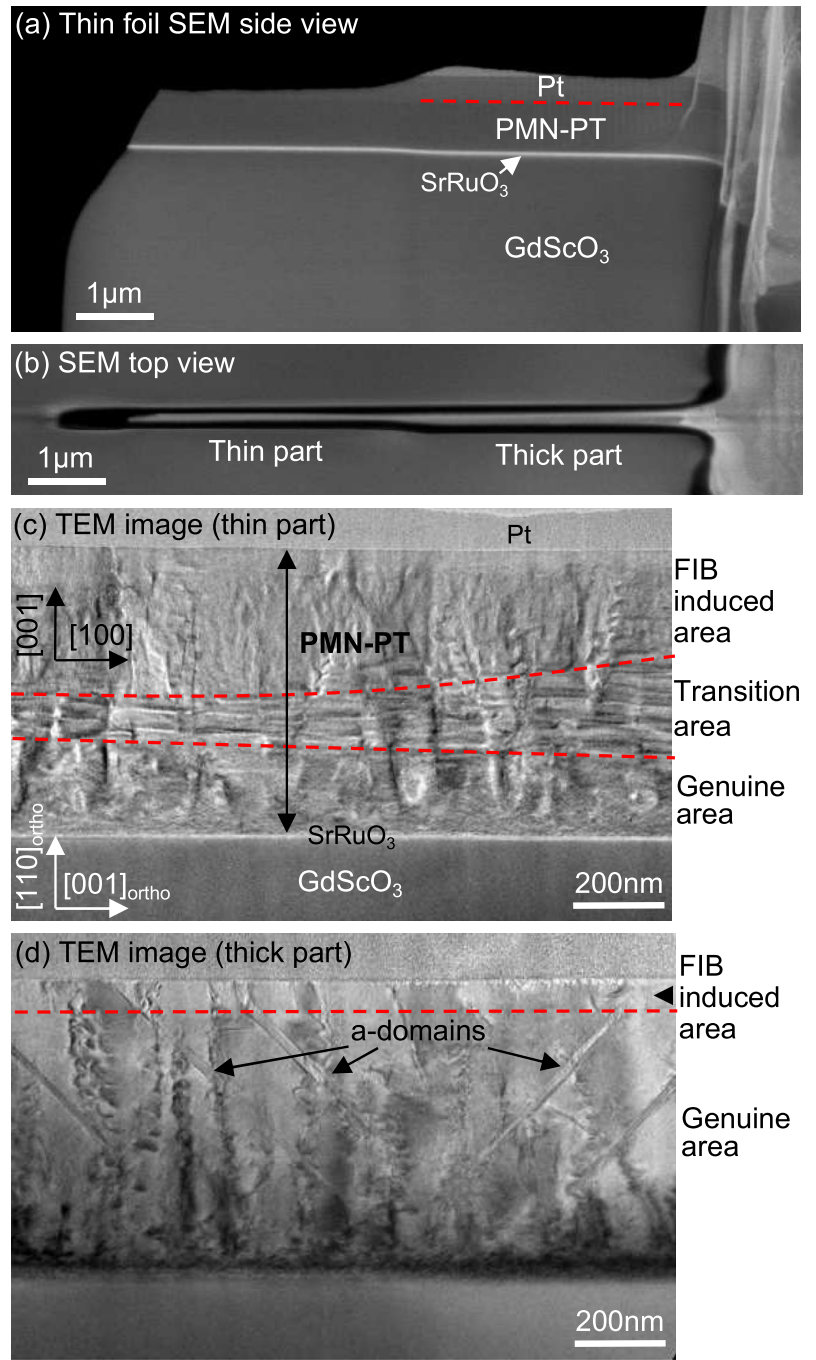

FIG. 1. (a), (b) SEM images of a FIB prepared lamella with a $600 \mathrm{~nm}$ thick layer of $0.4\left[\mathrm{~Pb}\left(\mathrm{Mg}_{1 / 3} \mathrm{Nb}_{2 / 3}\right) \mathrm{O}_{3}\right]-0.6\left[\mathrm{PbTiO}_{3}\right](0.4 \mathrm{PMN}-0.6 \mathrm{PT})$ grown by epitaxy on (110) $\mathrm{GdScO}_{3}$ substrate: (a) side-view and (b) top-view. The thickness of the lamella was estimated to be $80 \mathrm{~nm}$ and $140 \mathrm{~nm}( \pm 20 \mathrm{~nm})$ from the top-view image, in the thin and thick parts, respectively. (c), (d) TEM images of the (c) thin part and (d) thick part of the lamella showing distinct features. The thin part contains horizontal lines in the mid region. The thick part contains inclined needle-shaped $a$-domains (or $90^{\circ}$ domains).

extracted from the maps along the growth direction according to the dotted boxes.

In the first $250 \mathrm{~nm}$ above the interface, the $\varepsilon_{z z}$ deformation is high $(4.0 \% \pm 0.3 \%)$ and $\varepsilon_{x x}$ is low $(0.6 \% \pm 0.3 \%)$, which indicates that the $c$ axis is oriented in the growth direction (c-domain). In the transition area, between $250 \mathrm{~nm}$ and $450 \mathrm{~nm}, \varepsilon_{z z}$ decreases rapidly (down to $\approx 0.4 \%$ ), while $\varepsilon_{x x}$ stays constant. Beyond $450 \mathrm{~nm}$, in the FIB-induced area, both $\varepsilon_{z z}$ and $\varepsilon_{x x}$ remain small.

From the lattice parameters given in Section II, the two misfits between the PMN-PT and the GSO substrate are expected to be $\left(c_{\mathrm{PMN}-\mathrm{PT}}-d_{\mathrm{GSO}}^{(110)}\right) / d_{\mathrm{GSO}}^{(110)}=2.6 \%$ for the $c$ parameter of the PMN-PT and $\left(a_{\mathrm{PMN}-\mathrm{PT}}-d_{\mathrm{GSO}}^{(002)}\right) / d_{\mathrm{GSO}}^{(002)}=0.1 \%$ for the $a$ parameter, with $d_{\mathrm{GSO}}^{(110)}$ and $d_{\mathrm{GSO}}^{(002)}$ being the (110) and (002) orthorhombic distances of the GSO. The measured deformations are therefore larger than expected, which suggests a higher content in $\mathrm{PbTiO}_{3}$ than the nominal composition. 

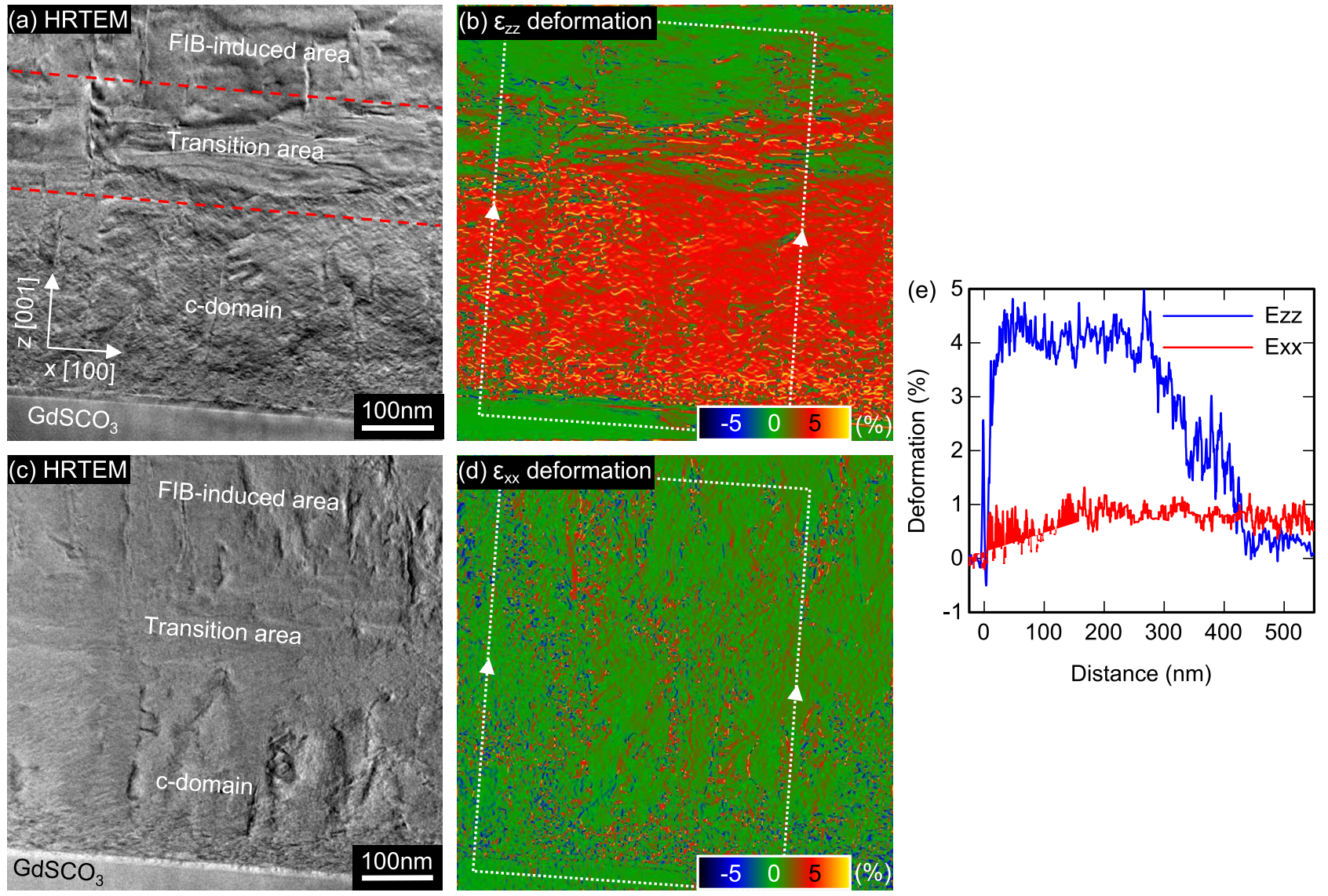

FIG. 2. (a) HRTEM image acquired in the thin part of the lamella. The sample was oriented along the (001) systematic row of the PMN-PT. (b) $\varepsilon_{z z}$ out-of-plane deformation map obtained by geometrical phase analysis. The spatial resolution defined by the size of the mask used in Fourier space is $6 \mathrm{~nm}$. $\varepsilon_{z z}$ is high $(4.0 \% \pm 0.3 \%)$ in the bottom region of the film and decreases strongly in the middle region (transition area). (c) HRTEM image of the same region acquired with the sample oriented along the (100) systematic row. (d) $\varepsilon_{x x}$ in-plane deformation map. $\varepsilon_{x x}$ remains low (0.6\% $\left.\pm 0.3 \%\right)$ over the whole image. (e) Average deformation profiles extracted from the maps (b) and (d) along the growth direction according to the dotted boxes.

Figs. 3(a)-3(d) show the same set of images as in Fig. 2 but, in the thick part of the lamella, near the surface of the film. Fig. 3(e) shows four deformation profiles extracted from the maps according to the numbered dotted boxes. Here, the deformation is defined relatively to the $c$-domains of the PMN-PT (the deformation is set to zero in the $c$ domains) because the substrate is not within the field of view.

The images contain two $a$-domains which are characterized by a decrease of $\varepsilon_{z z}$ and an increase of $\varepsilon_{x x}$ by about $3 \%$ (see profiles (1) and (3)). This is slightly larger than the variation calculated from the expected parameters: $\left(c_{\mathrm{PMN}-\mathrm{PT}}\right.$ $\left.-a_{\mathrm{PMN}-\mathrm{PT}}\right) / c_{\mathrm{PMN}-\mathrm{PT}}=2.5 \%$. In the FIB-induced area, $\varepsilon_{z z}$ is constant and is the same as in the $a$-domains: $-3.0 \% \pm 0.5 \%$ (see profile (2)). $\varepsilon_{x x}$ in the FIB-induced area is similar to the genuine area: $0.0 \%$ in the region located above the $c$-domains and $+3.0 \% \pm 0.5 \%$ above the $a$-domain (see profile (4)). However, the deformation at the domain walls is more diffuse and the domain walls are more vertical, i.e., along the [001] direction.

Therefore, the measurements allow us to create a representation of the domain structure shown in Fig. 3(f). In the genuine area, the $c$ parameter is always in the observation plane: vertical in the $c$-domains and horizontal in the $a$-domains. In the FIB-induced area, the $c$ parameter is measured only in the region located above the $a$-domains and is still horizontal. Above the $c$-domains, the $a$ parameter is in both directions of the observation plane, vertical and horizontal.

\section{DISCUSSION}

We have observed that the PMN-PT layer contains an area located near the surface, which is characterized by a decrease in the out-of-plane deformation. Our hypothesis, represented in Fig. 4, is that this area is induced by the preparation and corresponds to a flip of the crystal by $90^{\circ}$ around the horizontal axis, which allows the $c$ parameter to be in the thinning direction, i.e., perpendicular to the observation plane.

This hypothesis is supported by several observations. First, the size of this area depends on the thickness of the lamella (the size increases when the thickness of the lamella decreases) which indicates that this mechanism is related to the thinning procedure. Second, the horizontal lines in the thin part of the lamella and the deformation measurements indicate a structural variation in the vertical direction only. Third, the 2D deformation field measured in the thick part of the lamella changes in the $c$-domains but stays the same in the $a$-domains. In the $c$-domains, the unit cell rotates around 

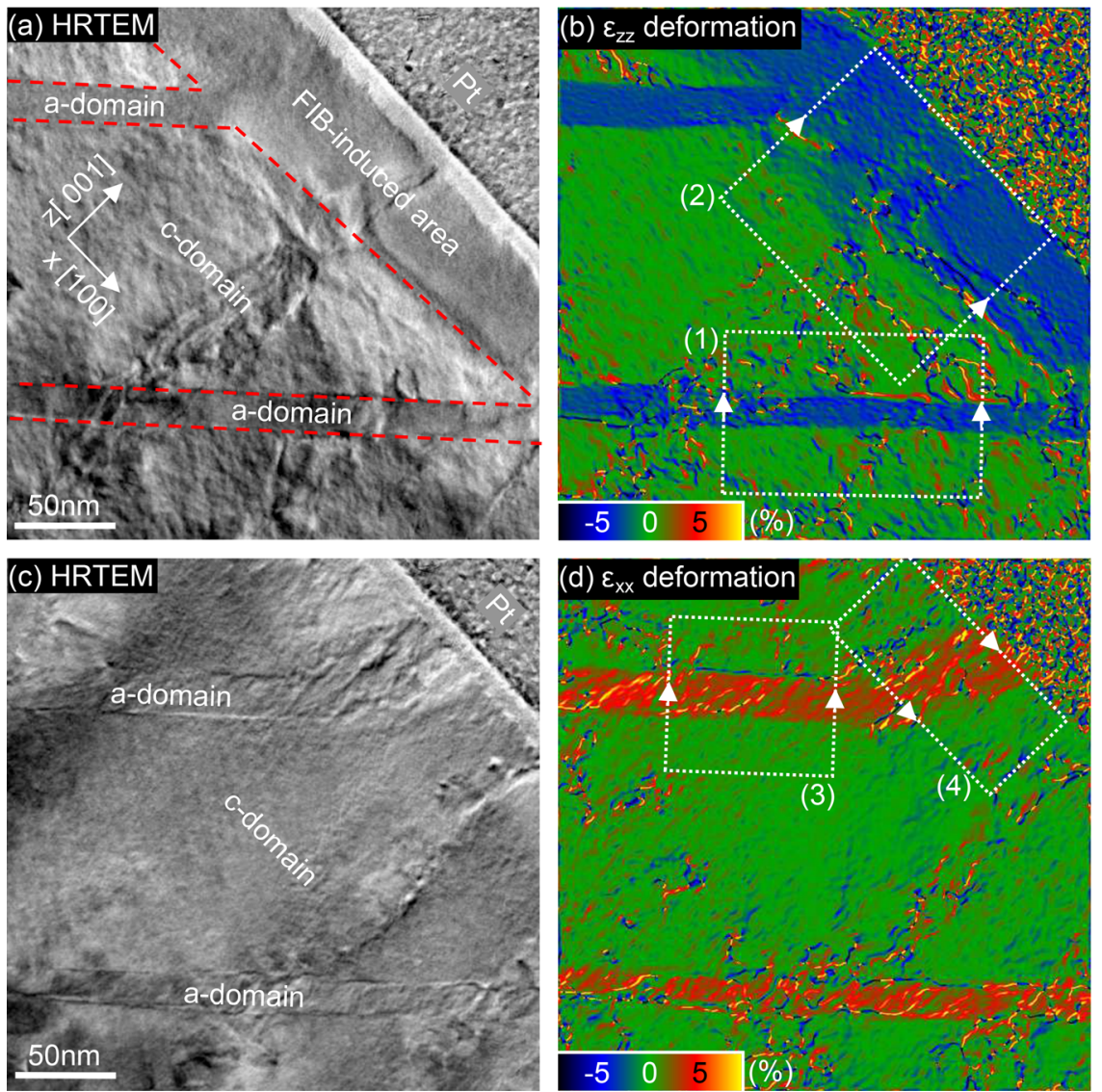

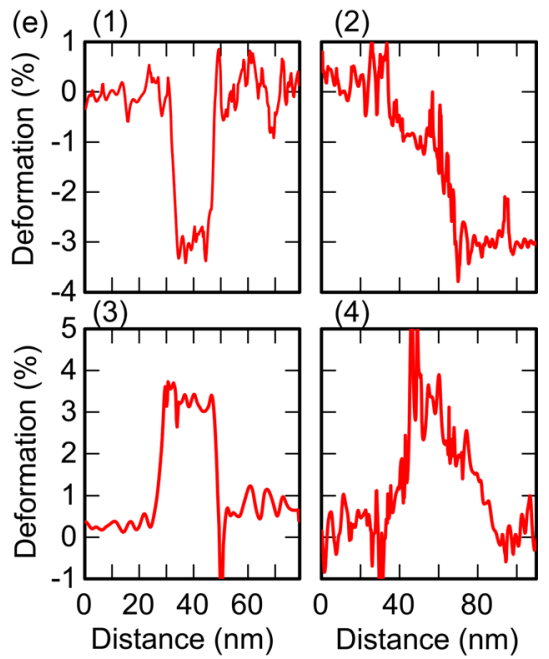

(f)

FIB-induced area

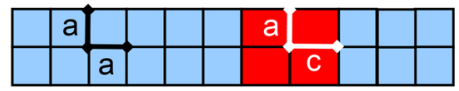

Genuine area

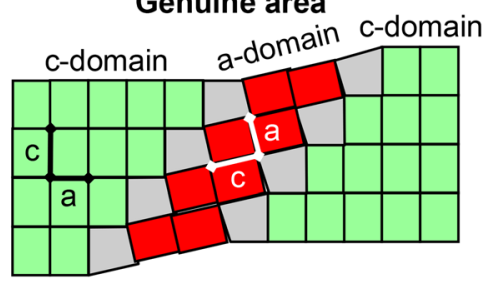

FIG. 3. (a) HRTEM image acquired in the thick part of the lamella near the surface of the layer. The sample was oriented along the (001) systematic row of the PMN-PT. (b) $\varepsilon_{z z}$ out-of-plane deformation map obtained by geometrical phase analysis. The deformation was set to zero in the $c$-domains. The spatial resolution defined by the size of the mask is $3 \mathrm{~nm} . \varepsilon_{z z}$ in the FIB-induced area is constant and is the same as in the $a$-domains: $-3.0 \% \pm 0.5 \%$. (c) HRTEM image acquired with the sample oriented along the (100) systematic row. (d) $\varepsilon_{x x}$ in-plane deformation map. $\varepsilon_{x x}$ in the FIB-induced area is similar to the genuine area: $0.0 \%$ in the region located above the $c$-domains and $+3.0 \% \pm 0.5 \%$ above the $a$-domain. (e) Average deformation profiles extracted from the maps (b) and (d) according to the numbered dotted boxes. (f) Representation of the lattice structure in the FIB-induced area and the genuine area. Above the $c$-domains, in the FIB-induced area, the $a$ parameter is in both directions of the observation plane, vertical, and horizontal. Above the $a$-domains, the parameters are the same as in the genuine area.

the $a$-axis, which is in the observation plane. The $c$ parameter is then flipped along the electron beam direction, and the $a$ parameter is present in both directions of the observation plane. In the $a$-domains, the unit cell flips around the $c$-axis which leads to a result equivalent to the initial state in the case of a tetragonal structure ( $a=b$, see Fig. 4). Fourth, the $a / c$ domain walls are not abrupt in the FIB-induced area because they are no more perpendicular to the observation direction but inclined by $45^{\circ}$. The projection of the information over the thickness of the lamella leads to a diffuse representation of the domain walls.

The reasons for the occurrence of this relaxation mechanism can be discussed, first, regarding the geometry of the lamella, and second, the influence of the ion beam.

We observed that this mechanism occurs preferentially in the top region of the layer, which can be explained in a similar way to the elastic relaxation in thin foils of epitaxial samples. Close to the bottom interface, a biaxial strain is induced by the substrate, which maintains the $c$-axis in the vertical direction. Near the surface, the sample is free to relax and the PMN-PT layer (600 nm thick) being thicker than the lamella ( 80 to $150 \mathrm{~nm}$ thick), the thin foil geometry

\section{FIB-induced area}

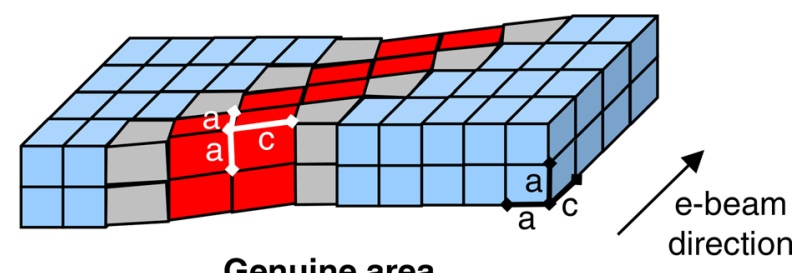

Genuine area

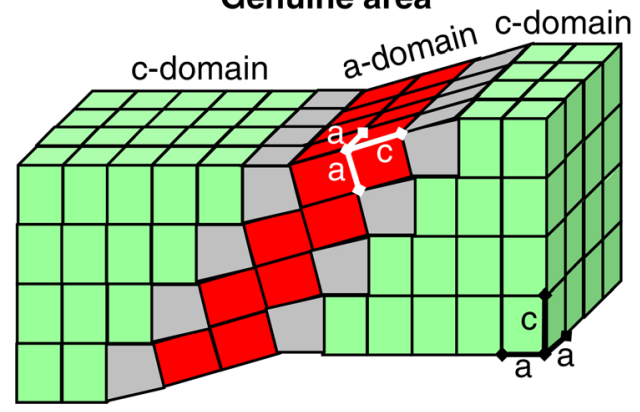

FIG. 4. Representation of the lattice structure in the genuine area and the FIB-induced area. The structure is flipped by $90^{\circ}$ in the FIB-induced area so that the $c$ parameter in the $c$-domains is oriented along the thinning direction (e-beam direction). For the $a$-domains, the $c$ parameter remains in-plane after rotation but the domain walls are inclined by $45^{\circ}$ relatively to the electron beam direction. 
can favor a structural relaxation in the direction perpendicular to the free surfaces.

Ion irradiation can lead to different phenomena, such as amorphization of the surfaces, formation of dislocations, interstitials, vacancies, and the local rise of temperature. ${ }^{5,28} \mathrm{The} \mathrm{Pt}$ capping layer being first deposited by electron beam assistance, we discuss only the possible damages related to the thinning, i.e., when scanning the ion beam on the sides of the lamella. In $\mathrm{Pb}(\mathrm{Zr}, \mathrm{Ti}) \mathrm{O}_{3}$, the implantation depth of $\mathrm{Ga}^{+}$ions on the sides of the lamella is $5 \mathrm{~nm}$ at $16 \mathrm{kV}$ and $2 \mathrm{~nm}$ at $5 \mathrm{kV} .{ }^{29}$ Assuming that $0.4 \mathrm{PMN}-0.6 \mathrm{PT}$ has a behavior similar to $\mathrm{Pb}(\mathrm{Zr}, \mathrm{Ti}) \mathrm{O}_{3}$ under the ion beam, the thickness of the implanted layer should be between 5 and $2 \mathrm{~nm}$ in our case, which is relatively small compared to the total thickness of the lamella. A large part of the lamella remains crystalline, which is supported by the high resolution images. Moreover, the deformation in the FIB-induced area is quite uniform. The crystalline quality is similar to the genuine area and shows no obvious signs of defects that could be induced by the ion beam, except in the transition zone between the genuine and FIB-induced areas where the defects are involved in the accommodation of the lattice reorientation. On the other hand, the rise of the temperature under the ion beam could favor the relaxation. It has been reported that the sample temperature under the ion milling can increase up to $300^{\circ} \mathrm{C}$ depending on the thermal properties of the material. ${ }^{30}$ The phase diagram of $0.4 \mathrm{PMN}-0.6 \mathrm{PT}$ shows that the tetragonal-cubic phase transition occurs at about $300{ }^{\circ} \mathrm{C} .{ }^{22}$ Even if the temperature is lower, heating could slightly change the lattice parameters and generate a biaxial strain. The proposed mechanism of structural reorientation might take place when cooling down, encouraged by the free surfaces. Alternatively, another source of stress is related to the amorphous layers. Micro-domains induced by the ion milling have been reported in 0.7PMN$0.3 \mathrm{PT}$, and their origins were attributed to the strain at the interface between the amorphous layers and the crystal. ${ }^{17}$ More investigations would be necessary to dissociate the influence of the ion beam conditions and the geometry of the lamella on the observed relaxation mechanism.

\section{CONCLUSION}

We have investigated the deformation in a $600 \mathrm{~nm}$ thick tetragonal PMN-PT epitaxial layer prepared by focused ion beam using geometrical phase analysis of HRTEM images. We have found that FIB preparation creates an area in the top part of the film where the out-of-plane deformation decreases strongly. The size of this area depends on the thickness of the lamella. The observations indicate that the structure flipped by $90^{\circ}$ so that the $c$-axis is in the thinning direction. Therefore, particular care must be taken for the preparation of this type of material. This relaxation mechanism could be investigated further using other preparation techniques (mechanical polishing, low energy ion milling) and phase field simulations ${ }^{31,32}$ to determine the critical parameters. For FIB preparation, it can be advised to prepare lamellae of different thicknesses to facilitate the identification of the relaxed areas. In the present case, relaxation was observed below $140 \mathrm{~nm}$ thick. Besides, we have found that the evaporation of a thick layer of platinum prior insertion in the FIB helps to prevent the apparition of these areas, where the crystal is reoriented. Finally, it can be advised to leave thick regions on both sides and the bottom part of the lamella to maintain a certain mechanical support.

\section{ACKNOWLEDGMENTS}

This work was funded through the European Metrology Research Programme (EMRP) Project IND54 Nanostrain. The EMRP is jointly funded by the EMRP participating countries within EURAMET and the European Union. The authors acknowledge the European Union under the Seventh Framework Programme under a contract for an Integrated Infrastructure Initiative Reference 312483-ESTEEM2. The authors acknowledge the "Conseil Regional Midi-Pyrénées" and the European FEDER for financial support within the CPER program. This work has been supported by the French National Research Agency under the "Investissement d'Avenir" Program Reference No. ANR-10-EQPX-38-01. The work at the University of Wisconsin-Madison was supported by the Army Research Office through Grant No. N00014-07-1-0215.

${ }^{1}$ L. A. Giannuzzi and F. A. Stevie, Micron 30, 197 (1999).

${ }^{2}$ J. Ayache and P. H. Albarède, Ultramicroscopy 60, 195 (1995).

${ }^{3}$ J. McCaffrey, Ultramicroscopy 38, 149 (1991).

${ }^{4}$ R. M. Langford and A. K. Petford-Long, J. Vac. Sci. Technol., A 19, 2186 (2001).

${ }^{5}$ J. Mayer, L. A. Giannuzzi, T. Kamino, and J. Michael, MRS Bull. 32, 400 (2007).

${ }^{6}$ J. M. Gibson, R. Hull, J. C. Bean, and M. M. J. Treacy, Appl. Phys. Lett. 46, 649 (1985).

${ }^{7}$ M. M. J. Treacy and J. M. Gibson, J. Vac. Sci. Technol. B 4, 1458 (1986).

${ }^{8}$ D. D. Perovic, G. C. Weatherly, and D. C. Houghton, Philos. Mag. A 64, 1 (1991).

${ }^{9}$ L. Clement, R. Pantel, L. F. T. Kwakman, and J. L. Rouviere, Appl. Phys. Lett. 85, 651 (2004)

${ }^{10}$ F. Houdellier, C. Roucau, L. Clément, J. Rouvière, and M. Casanove, Ultramicroscopy 106, 951 (2006).

${ }^{11}$ K. Tillmann, M. Lentzen, and R. Rosenfeld, Ultramicroscopy 83, 111 (2000).

${ }^{12}$ M. Hÿtch, E. Snoeck, and R. Kilaas, Ultramicroscopy 74, 131 (1998).

${ }^{13}$ S.-E. Park and T. R. Shrout, J. Appl. Phys. 82, 1804 (1997).

${ }^{14}$ Q. Zhou, X. Xu, E. Gottlieb, L. Sun, J. Cannata, H. Ameri, M. Humayun, P. Han, and K. Shung, IEEE Trans. Ultrason., Ferroelectr., Freq. Control 54, 668 (2007).

${ }^{15}$ D. Newns, B. Elmegreen, X. Hu Liu, and G. Martyna, J. Appl. Phys. 111, 084509 (2012).

${ }^{16}$ P. M. Solomon, B. A. Bryce, M. A. Kuroda, R. Keech, S. Shetty, T. M. Shaw, M. Copel, L.-W. Hung, A. G. Schrott, C. Armstrong, M. S. Gordon, K. B. Reuter, T. N. Theis, W. Haensch, S. M. Rossnagel, H. Miyazoe, B. G. Elmegreen, X.-H. Liu, S. Trolier-McKinstry, G. J. Martyna, and D. M. Newns, Nano Lett. 15, 2391 (2015).

${ }^{17}$ K.-H. Kim, D. A. Payne, and J.-M. Zuo, Appl. Phys. Lett. 97, 261910 (2010).

${ }^{18}$ V. Srot, M. Gec, P. A. van Aken, J.-H. Jeon, and M. Ceh, Micron 62, 37 (2014).

${ }^{19}$ C. B. Eom, J. Z. Sun, K. Yamamoto, A. F. Marshall, K. E. Luther, T. H. Geballe, and S. S. Laderman, Appl. Phys. Lett. 55, 595 (1989).

${ }^{20}$ C. B. Eom, R. J. Cava, R. M. Fleming, J. M. Phillips, R. B. vanDover, J. H. Marshall, J. W. P. Hsu, J. J. Krajewski, and W. F. Peck, Science 258, 1766 (1992)

${ }^{21}$ B. Noheda, D. E. Cox, G. Shirane, J. Gao, and Z.-G. Ye, Phys. Rev. B 66, 054104 (2002).

${ }^{22} \mathrm{~A}$. Slodczyk, "Structural, dielectric and vibrational studies of lead magnesium niobate - lead titanate $(1-\mathrm{x}) \mathrm{PbMg}_{1 / 3} \mathrm{Nb}_{2 / 3} \mathrm{O}_{3}-\mathrm{xPbTiO}_{3}$ solid solutions," Ph.D. thesis (University of Silesia, 2006). 
${ }^{23}$ B. Velickov, V. Kahlenberg, R. Bertram, and M. Bernhagen, Z. Kristallogr. 222, 466 (2007).

${ }^{24}$ C. W. Jones, P. D. Battle, P. Lightfoot, and W. T. A. Harrison, Acta Crystallogr., Sect. C 45, 365 (1989).

${ }^{25}$ F. Hüe, C. L. Johnson, S. Lartigue-Korinek, G. Wang, P. R. Buseck, and M. J. Hÿtch, J. Electron Microsc. 54, 181 (2005).

${ }^{26}$ C. S. Ganpule, V. Nagarajan, H. Li, A. S. Ogale, D. E. Steinhauer, S. Aggarwal, E. Williams, R. Ramesh, and P. De Wolf, Appl. Phys. Lett. 77, $292(2000)$
${ }^{27}$ I. Vrejoiu, Y. Zhu, G. Le Rhun, M. A. Schubert, D. Hesse, and M. Alexe, Appl. Phys. Lett. 90, 072909 (2007).

${ }^{28}$ S. Rubanov and P. Munroe, Microsc. Microanal. 11, 446 (2005).

${ }^{29}$ N. Wollschläger, W. Österle, I. Häusler, and M. Stewart, Phys. Status Solidi C 12, 314 (2015).

${ }^{30}$ Y. M. Park, D.-S. Ko, K.-W. Yi, I. Petrov, and Y.-W. Kim, Ultramicroscopy 107, 663 (2007).

${ }^{31}$ Y. L. Li, S. Y. Hu, Z. K. Liu, and L. Q. Chen, Appl. Phys. Lett. 78, 3878 (2001).

${ }^{32}$ J. Britson, C. Nelson, X. Pan, and L.-Q. Chen, Acta Mater. 75, 188 (2014). 